\title{
Microstrip Bandstop Filter for Preventing Conduction Electromagnetic Information Leakage of High-Power Transmission Line
}

\author{
Xueyun Han $\mathbb{D}^{1,2}$ Xiaosong Li, ${ }^{1}$ Yingping Zhou, ${ }^{1}$ Pengfei Zheng, ${ }^{1}$ Haohua Cui, ${ }^{2}$ \\ and Xiaonan Zhang ${ }^{2}$ \\ ${ }^{1}$ College of Electronic and Electrical Engineering, Henan Normal University, Xinxiang, China \\ ${ }^{2}$ Henan Key Laboratory of Optoelectronic Sensing Integrated Application, Xinxiang, China \\ Correspondence should be addressed to Xueyun Han; hanxueyun126@126.com
}

Received 7 June 2021; Revised 5 January 2022; Accepted 29 January 2022; Published 18 February 2022

Academic Editor: Anna Pietrenko-Dabrowska

Copyright (C) 2022 Xueyun Han et al. This is an open access article distributed under the Creative Commons Attribution License, which permits unrestricted use, distribution, and reproduction in any medium, provided the original work is properly cited.

\begin{abstract}
The research of transient electromagnetic pulse emission monitoring technology (TEMPEST) protection is essential. Based on microstrip bandstop filter (MSBSF), a method to prevent electromagnetic information leakage of high-power transmission lines is proposed in this paper. The MSBSF with a high insertion loss and carrying large current in the frequency of $2.40-2.49 \mathrm{GHz}$ is designed, fabricated, and experimentally measured. The fabricated MSBSF with the insertion loss of $38 \mathrm{~dB}$ at $2.41-2.49 \mathrm{GHz}$ can carry a current greater than $10 \mathrm{~A}$ and withstand a voltage of $1.7 \mathrm{kV}$. Compared with the traditional electromagnetic interference (EMI) filter, the MSBSF has the advantages of preventing conducted electromagnetic information leakage in the high-frequency bands, carrying greater current subject to higher voltage, and possessing lighter weight. The MSBSF may be used to prevent the leakage of high-frequency electromagnetic information of high-power transmission lines.
\end{abstract}

\section{Introduction}

With the rapid development of information and communication technology, electromagnetic information leakage associated with telecommunication devices, such as transmission lines, brings increasingly strong impacts on information security. Transient electromagnetic pulse emission monitoring technology (TEMPEST) is known for using reverse technology to collect and restore the transmitting source signals and preventing potential security threats of information equipment [1]. Nowadays, many scholars have begun to actively research and develop TEMPEST [2-6], especially TEMPEST protection. Many methods of TEMPEST protection have been achieved, for example, low-radiation equipment [7-10], noise interference [11], electromagnetic shielding [12-14], filtering technology [15], and optical fiber technology [16, 17]. Low-radiation equipment, noise interference, electromagnetic shielding, and optical fiber technology mainly prevent electromagnetic information leakage caused by electromagnetic radiation. Filtering technology is the only way to prevent electromagnetic information leakage caused by conduction.

With the development of high-speed communication technology, the leakage of electromagnetic information in high-frequency band should be widely concerned. In 2005, the electromagnetic information leakage up to $3 \mathrm{GHz}$ was measured by K. Hoffmann and Skvor [18]. Traditional electromagnetic interference (EMI) filters at their operation frequency can block information only in the frequency range of $150 \mathrm{KHz}-30 \mathrm{MHz}$ and carry a current of $10 \mathrm{~A}$ and withstand a voltage of $250 \mathrm{~V}$. What is worse, EMI filter designed by using lumped components has greater volume and weight and cannot prevent information leakage of information equipment in high-frequency band $(\sim \mathrm{GHz})$.

The microstrip filter made of PCB has the advantages of light weight and small volume, which has been studied by many scholars. In addition, based on a triple path signal interference mechanism, Song et al. [19] proposed a compact 
broadband bandstop filter with the insertion loss of $30 \mathrm{~dB}$ for use in modern wireless systems. A broadband commonmode filter with the insertion loss of $10 \mathrm{~dB}$ was designed for solving electromagnetic interference in high-speed differential digital systems and discussed by Chan et al. [20]. Phudpong and Hunter [21] projected a prototype of a singleresonator bandstop limiter with an insertion loss of $32 \mathrm{~dB}$, a resonant frequency of $2 \mathrm{GHz}$, and a limiting bandwidth of approximately $200 \mathrm{MHz}$. The dual-wideband bandstop filters, which are used in multi-wideband wireless systems, were demonstrated by Feng et al. [22]. A planar high insertion loss bandstop filter within a frequency band from 2.79 to $3.21 \mathrm{GHz}$ was developed by $\mathrm{Wu}$ et al. [23]. However, those microstrip filters are used in low-power communication circuits and devices. To the authors' best knowledge, microstrip filters for preventing conduction electromagnetic information leakage of high-power transmission lines are rarely reported.

Because of the importance of TEMPEST protection, a method to prevent electromagnetic information leakage of high-power transmission lines based on the microstrip bandstop filter (MSBSF) is proposed in this paper. The MSBSF with high insertion loss at $2.40-2.49 \mathrm{GHz}$ is designed, fabricated, and measured. The results show that it can carry the current greater than $10 \mathrm{~A}$, withstand the voltage of $1.7 \mathrm{kV}$, and have the insertion loss of $38 \mathrm{~dB}$ at 2.41-2.49 GHz. Compared with the EMI filter, the MSBSF can more effectively prevent the conduction electromagnetic information leakage of high-power transmission lines in high-frequency band apart from carrying greater current subject to higher voltage, having lighter weight and smaller volume. The MSBSF has a good application in preventing conducted electromagnetic information leakage in high frequency.

\section{Design Ideas}

2.1. Schematic. As shown in Figure 1, when transmitting electromagnetic signal or energy over a long distance, the transmission line of an information device may carry electromagnetic information, and the information leakage will be caused by the process of probe detection and spectrum analysis. To prevent information leakage, the filter should be connected before the information device is connected to the transmission line.

2.2. Theory Analysis. Transmission lines of information devices such as power lines and telephone lines can be regarded as parallel two conductors because the length of transmission line is very long. In the situation of transmitting high-frequency electromagnetic waves, Kirchhoff's law cannot be applied to the calculation of voltage and current on a transmission line. Transmission line is divided into many very short transmission lines, the length of each segment is shorter than the wavelength of high-frequency electromagnetic waves, and Kirchhoff's law can be used for each segment of the transmission line. As shown in Figure 2, each segment of the transmission line can be equivalent to loss resistance, distributed inductance, conductivity, and distributed capacitance due to the electromagnetic coupling effect. The resistance $R_{0}$, inductance $L_{0}$, capacitance $C_{0}$, and conductance $G_{0}$ are denoted as

$$
\begin{aligned}
& R=\frac{2}{\pi d} \sqrt{\frac{\omega \mu 1}{2 \sigma 1}}, \\
& L=\frac{\mu}{\pi} \ln \frac{D+\sqrt{D^{2}-d^{2}}}{d}, \\
& C=\frac{\pi \varepsilon 1}{\ln D+\sqrt{D^{2}+d^{2}} / d} \\
& G=\frac{\pi \sigma}{\ln D+\sqrt{D^{2}+d^{2}} / d},
\end{aligned}
$$

where $\mu_{1}$ is the permeability of two conductors, $\sigma_{1}$ is the conductivity of two conductors, $\varepsilon_{1}$ is the dielectric constant of the medium between two conductors, $\mu$ is the permeability of the medium between two conductors, $\sigma$ is the conductivity of the medium between two conductors, $D$ is the distance between two conductors, and $d$ is the diameter of the two conductors.

Based on Kirchhoff's low, each segment transmission line is expressed as

$$
\begin{gathered}
\frac{\partial u(x, t)}{\partial x}+L \frac{\partial i(x, t)}{\partial t}+R i(x, t)=0, \\
\frac{\partial i(x, t)}{\partial x}+C \frac{\partial u(x, t)}{\partial t}+G u(x, t)=0,
\end{gathered}
$$

where $u(x, t)$ is the voltage at time $t$ and position $x$ on the transmission line; $i(x, t)$ is the current at time $t$ and position $x$ on the transmission line. When $u(x, t)$ and $i(x, t)$ change as a sine function of time, $u(x, t)$ and $i(x, t)$ can be expressed as

$$
\begin{aligned}
u(x, t) & =\mathrm{u} \max \cos (w t+y u(x)) \\
& =\mathrm{u} \max e^{i \omega t} e^{y u(x)} \\
& =u(x) e^{i \omega t}, \\
i(x, t) & =\mathrm{i} \max \cos (w t+y i(x)) \\
& =\mathrm{i} \max e^{i \omega t} e^{y i(x)} \\
& =i(x) e^{i \omega t},
\end{aligned}
$$

where $u(x)$ is the voltage at position $x$ on the transmission line; $i(x)$ is the current at the position $x$ on the transmission line.

Equations (6) and (7) can be derived from the combined equations (2)-(5).

$$
\begin{aligned}
& \frac{\mathrm{d} u(x)}{\mathrm{d} x}=-(j \omega L+R) i(x), \\
& \frac{d i(x)}{d x}=-(j \omega C+G) u(x) .
\end{aligned}
$$

The derivative of equations (6) and (7) with respect to $x$, respectively, gives us the following: 


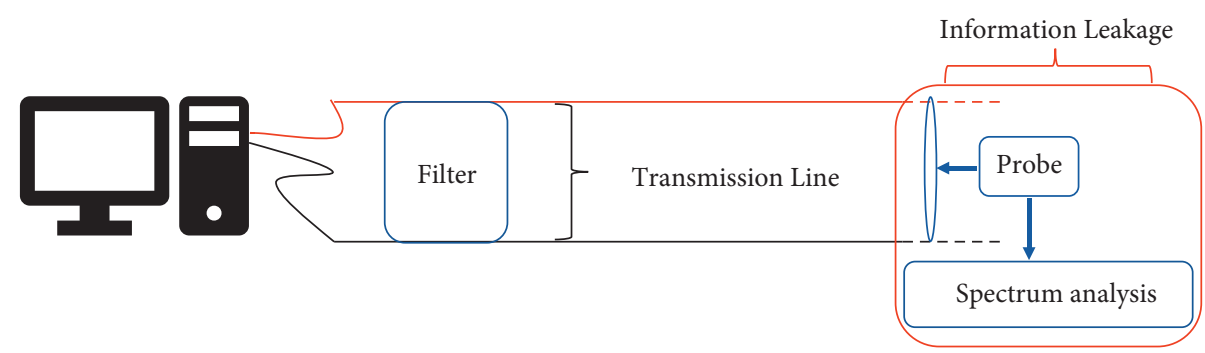

FIGURE 1: Schematic diagram of design ideas.

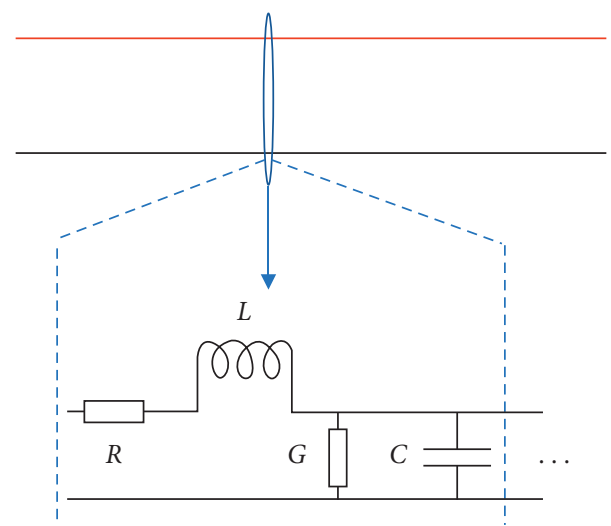

FIGURE 2: Equivalent circuit of the transmission line.

$$
\begin{aligned}
-\frac{d^{2} u(x)}{\mathrm{d} x^{2}} & =(j \omega L+R)(j \omega C+G) u(x) \\
& =\gamma^{2} u(x), \\
-\frac{d^{2} i(x)}{\mathrm{d} x^{2}} & =(j \omega L+R)(j \omega C+G) i(x) \\
& =\gamma^{2} i(x),
\end{aligned}
$$

where $\gamma$ is the propagation coefficient of voltage and current on the transmission line. By solving (8) and (9), the general solutions (10) and (11) can be obtained.

$$
\begin{aligned}
u(x) & =A 1 e^{\gamma x}+A 2 e^{\gamma x}, \\
i(x) & =\sqrt{\frac{G+j \omega L}{R+j \omega C}}\left(A 1 e^{-\gamma x}+A 2 e^{-\gamma x}\right) .
\end{aligned}
$$

When the voltage and current at $x=0$ on the transmission line are known, the voltage and current at any part of the transmission line can be obtained. The equation is described as follows:

$$
\begin{aligned}
& u(x)=\frac{1}{2}\left(u(0)+\sqrt{\frac{R+j \omega L}{G+j \omega C}} i(0)\right) e^{-\gamma x}+\frac{1}{2}\left(u(0)-\sqrt{\frac{R+j \omega L}{G+j \omega C}} i(0)\right) e^{\gamma x}, \\
& i(x)=\frac{1}{2}\left(\sqrt{\frac{G+j \omega C}{R+j \omega L}} u(0)+i(0)\right) e^{-\gamma x}-\frac{1}{2}\left(\sqrt{\frac{G+j \omega C}{R+j \omega L}} u(0)-i(0)\right) e^{\gamma x} .
\end{aligned}
$$

When the voltage or current on the transmission line is detected by a probe, information leakage will occur.

The schematic diagram of the filter is shown in Figure 3. It can be found that filter is a two-port network, the input voltage and current of port 1 are $u_{1}$ and $i_{1}$, respectively, and the output voltage and current of port 2 are $u_{2}$ and $i_{2}$, respectively. When the filter ports match, the forward voltage transmission of the filter can be expressed as [24]

$$
S_{21}=\frac{u_{2}-i_{2} Z_{0}}{u_{1}+i_{1} Z_{0}} .
$$

When the filter is a bandstop filter, the filter can reduce the voltage and current containing information on the 


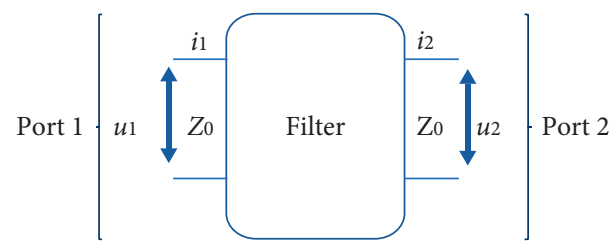

FIgURE 3: Schematic diagram of the filter.

transmission line. Hence, the bandstop filter can prevent information leakage before the information device is connected to the transmission line.

Based on the previously mentioned analysis, the bandstop filter is important to prevent the transmission line conducting electromagnetic information leakage. Therefore, it is worthy to research bandstop filter and the design of bandstop filter is mainly introduced in the following paper.

\section{Filter Structure}

The structure diagram of the traditional bandstop filter is shown in Figure 4(a). It is composed of connected and open stubs. The filter with connected and open stubs can achieve high insertion loss, but the physical width of connected stubs is too narrow to carry a high current. According to [23], the structure of the traditional bandstop filter can be transformed into that of Figure 4(c) by using the structure of Figure 4(b), and the unequal width of the coupled structure can be used in the structure of Figure 4(c), which enables the connected stubs to carry a high current. Hence, Figure 4(c) is the structure of the proposed MSBSF for preventing conduction electromagnetic information leakage of highpower transmission line in this paper.

As is shown in Figure 4(c), the proposed MSBSF consists of $n$ parts, and each part consists of a parallel coupling line with an open stub. To realize a high insertion loss $(>40 \mathrm{~dB})$ and has a simple structure, the MSBSF consists of only five parts. The reason MSBSF consists of only five parts is shown in Figure 5. It can be found that the MSBSF consists of only part1, or part $1+$ part2, or part $1+$ part $2+$ part 3 , or part $1+$ part 2 + part 3 + part 4 cannot realize high insertion loss, so the structure of the filter is composed of five parts, and the structure diagram in shown in Figure 2(d). The odd and even mode characteristic impedances of the parallel coupling lines are $Z_{\mathrm{o} 1}$ and $Z_{\mathrm{e} 1}, Z_{\mathrm{o} 2}$ and $Z_{\mathrm{e} 2}, Z_{\mathrm{o} 3}$ and $Z_{\mathrm{e} 3}, Z_{\mathrm{o} 4}$ and $Z_{\mathrm{e} 4}, Z_{\mathrm{o} 5}$ and $Z_{\mathrm{e} 5}$, respectively. The characteristic impedances of the open stubs are $Z_{1}, Z_{2}, Z_{3}, Z_{4}, Z_{5}$, respectively. The characteristic impedances of the port are $Z_{0}, Z_{0}=50 \Omega$. The electrical lengths of the parallel coupling lines and open stubs all are $\theta . \theta=\pi / 2$ at the center frequency of the stopband. The MSBSF is symmetrical around part 3 for the convenience of design and analysis. Because the MSBSF is symmetrical around part $3, Z_{1}=Z_{5}, Z_{2}=Z_{4}, Z_{\mathrm{o} 1}=Z_{\mathrm{o} 5}$, $Z_{\mathrm{e} 1}=Z_{\mathrm{e} 5}, Z_{\mathrm{o} 2}=Z_{\mathrm{o} 4}$, and $Z_{\mathrm{e} 2}=Z_{\mathrm{e} 4}$.

\section{Theory and Simulation Analysis}

4.1. Theory Analysis. According to the structure diagram of the MSBSF with five parts, the ABCD matrix of each part can be derived as [21]

$$
\left[\begin{array}{cc}
A_{i} & B_{i} \\
C_{i} & D_{i}
\end{array}\right]=\left[\begin{array}{cc}
\frac{\cos \theta}{k_{i}}+\frac{k_{i}\left(Z_{\mathrm{e} i}+Z_{\mathrm{o} i}\right) \cos \theta-\left(Z_{\mathrm{e} i}-Z_{\mathrm{o} i}\right)}{2 Z_{i}} \mathrm{j} \frac{\left(Z_{\mathrm{e} i}-Z_{\mathrm{o} i}\right)-k i\left(Z_{\mathrm{e} i}+Z_{\mathrm{o} i}\right) \cos ^{2} \theta}{2 \sin \theta} \\
\mathrm{j} \frac{2 \sin \theta}{Z_{\mathrm{e} i}-Z_{\mathrm{o} i}}+\mathrm{j} \frac{\sin \theta}{k_{i} Z_{i}} & \frac{\cos \theta}{k_{i}}
\end{array}\right]
$$

where $k_{i}$ is the coupling coefficient and is defined as (19), $i=1,2,3,4,5$.

$$
k \mathrm{i}=\frac{Z_{\mathrm{e} i}-Z_{\mathrm{o} i}}{Z_{\mathrm{e} i}+Z_{\mathrm{o} i}}
$$

Since each part is connected in cascade, the ABCD matrix of MSBSF can be described as

$$
\left[\begin{array}{ll}
A & B \\
C & D
\end{array}\right]=\left[\begin{array}{ll}
A_{1} & B_{1} \\
C_{1} & D_{1}
\end{array}\right] \times\left[\begin{array}{ll}
A_{2} & B_{2} \\
C_{2} & D_{2}
\end{array}\right] \times\left[\begin{array}{cc}
A_{3} & B_{3} \\
C_{3} & D_{3}
\end{array}\right] \times\left[\begin{array}{cc}
A_{4} & B_{4} \\
C_{4} & D_{4}
\end{array}\right] \times\left[\begin{array}{cc}
A_{5} & B_{5} \\
C_{5} & D_{5}
\end{array}\right]
$$

Based on formula (17), when $S_{21}=0$, the transmission zeros of MSBSF can be calculated.

$$
S_{21}=\frac{2}{A+B Y_{0}+C Z_{0}+D}
$$

4.2. Simulation Analysis. The influence of MSBSF's characteristic impedance on its insertion loss and stopband bandwidth is explored by using HFSS (3D electromagnetic simulation software). By simulation, the results are shown in Figure 6(a), and it can be found that the insertion loss 


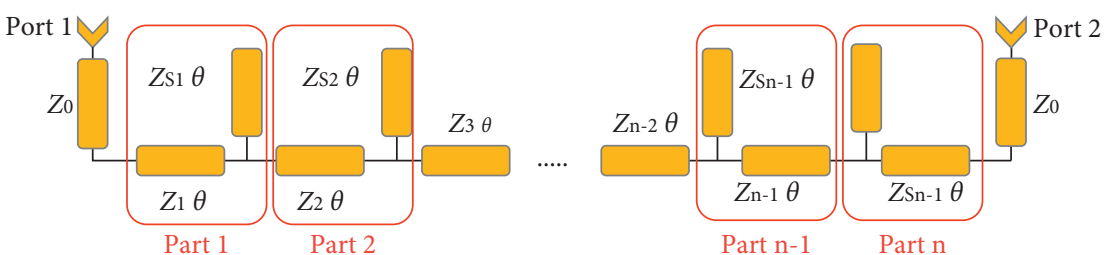

(a)

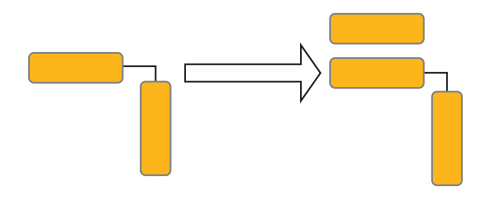

(b)

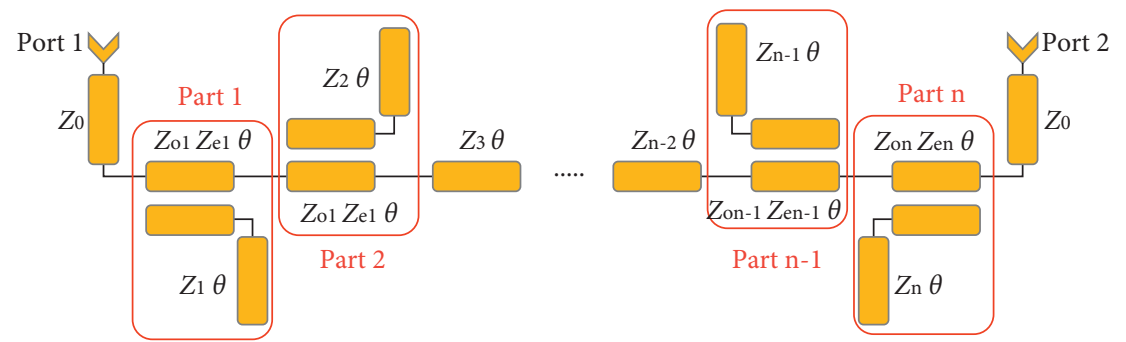

(c)

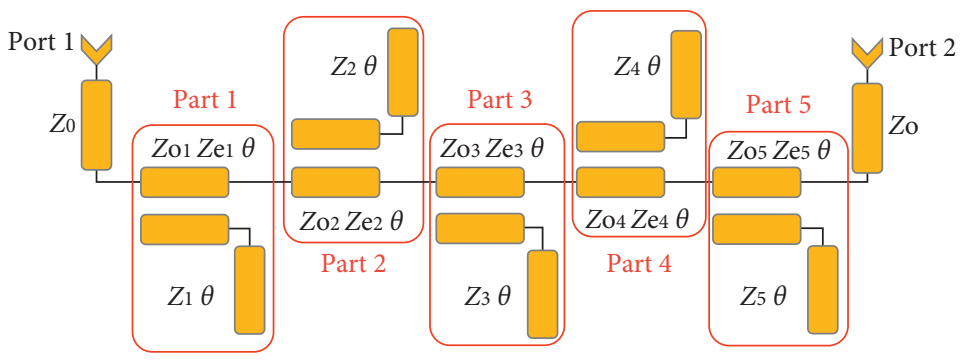

(d)

FIGURE 4: Structure diagram: (a) traditional bandstop filter with multiple parts; (b) equivalent structure; (c) proposed bandstop filter with multiple parts; (d) bandstop filter with five parts.

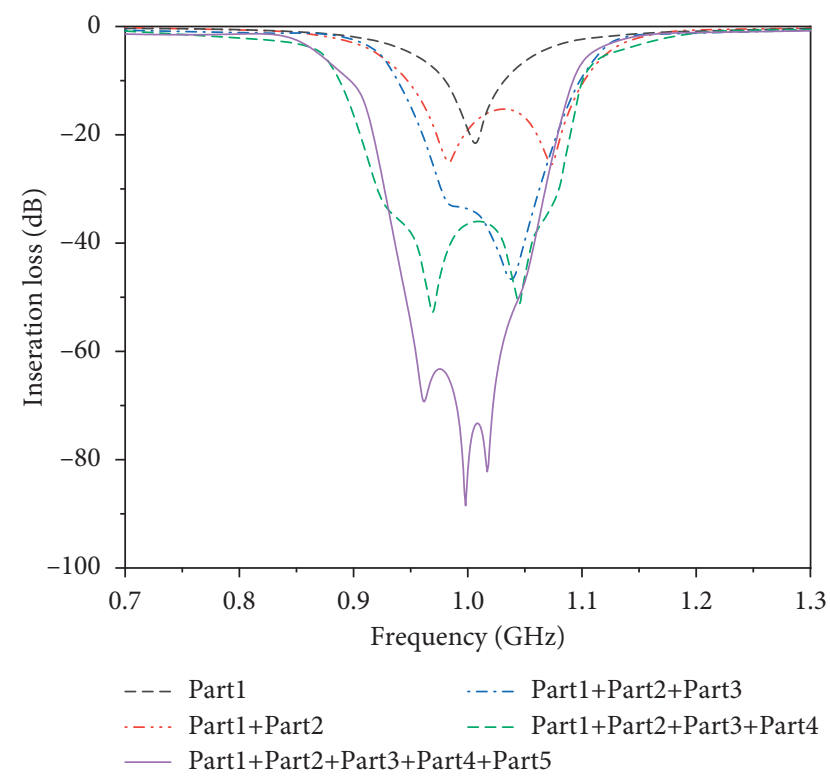

FIGURE 5: Filtering performance of the proposed bandstop filter.

increases with the increase of the characteristic impedance of $Z_{1}\left(Z_{5}\right)$ and $Z_{3}$, and decreases with the increase of the characteristic impedance of $Z_{2}\left(Z_{4}\right)$. When $Z_{1}\left(Z_{5}\right)$ is greater than $90 \Omega, Z_{2}\left(Z_{4}\right)$ is less than $80 \Omega$, and $Z_{3}$ is greater than $80 \Omega$, the insertion loss of MSBSF is greater than $50 \mathrm{~dB}$. The relationship between $Z_{1}\left(Z_{5}\right), Z_{2}\left(Z_{4}\right), Z_{3}$, and bandwidth at an insertion loss of $50 \mathrm{~dB}$ is shown in Figure 6(b), respectively. When the insertion loss is greater than $50 \mathrm{~dB}$, the bandwidth at $50 \mathrm{~dB}$ decreases with the increase of characteristic impedance $Z_{1}\left(Z_{5}\right)$ and $Z_{3}$ and increases with the increase of characteristic impedance $Z_{2}\left(Z_{4}\right)$.

Figure 7 (a) shows the relationship between the coupling coefficient and maximum insertion loss. It can be found that when coupling coefficient $k_{1}\left(k_{5}\right)$ is greater than 0.45 , the insertion loss of MSBSF is greater than $50 \mathrm{~dB}$, and when the coupling coefficients $k_{2}\left(k_{4}\right)$ and $k_{3}$ are 0.3 to 0.45 , the insertion loss is greater than $50 \mathrm{~dB}$. When the insertion loss is greater than $50 \mathrm{~dB}$, the relationship between the coupling coefficient and the bandwidth at insertion loss $50 \mathrm{~dB}$ is shown in Figure 7(b). It can be found that the bandwidth at insertion loss $50 \mathrm{~dB}$ increases with the increase of the coupling coefficient of $k_{2}\left(k_{4}\right)$ and $k_{3}$ and decreases with the increase of the characteristic impedance of $k_{1}\left(k_{5}\right)$.

Through the previously mentioned simulation analysis, the design process of the MSBSF can be summarized as follows:

(1) Specify the insertion loss and the bandwidth of the designed MSBSF

(2) According to Figures 6 and 7, the coupling coefficient and characteristic impedance are determined

(3) Finally, optimization is done using HFSS 


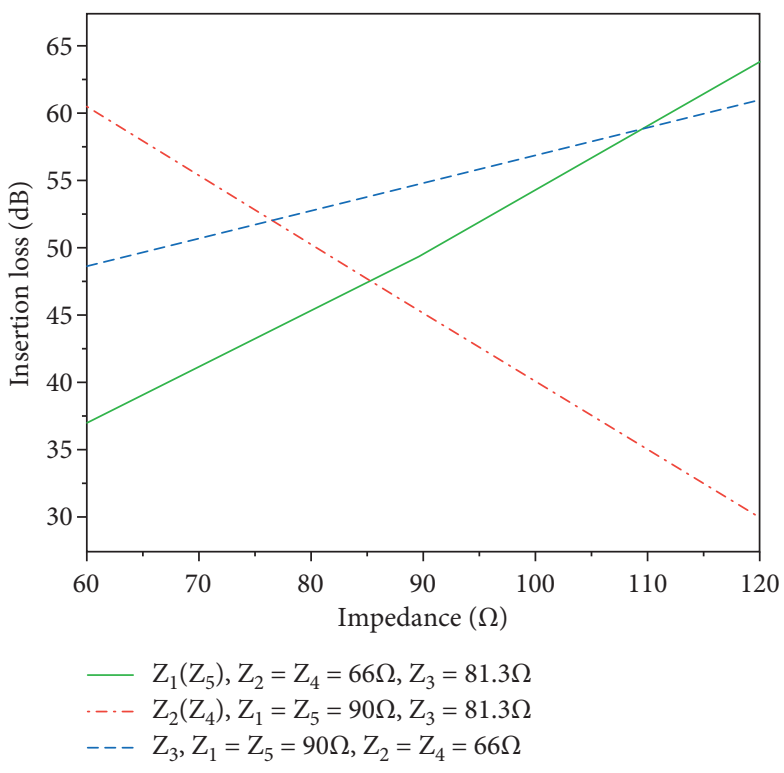

(a)

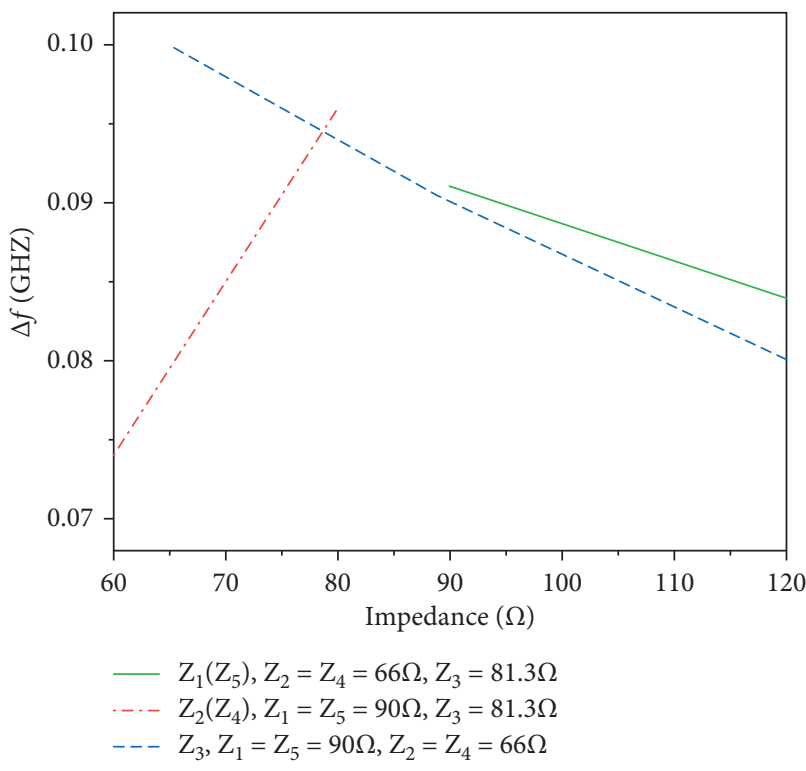

(b)

Figure 6: Simulation analysis: (a) the relationship between $Z_{1}\left(Z_{5}\right), Z_{2}\left(Z_{4}\right), Z_{3}$ and maximum insertion loss; (b) the relationship between $Z_{1}\left(Z_{5}\right), Z_{2}\left(Z_{4}\right), Z_{3}$ and bandwidth at insertion loss $50 \mathrm{~dB} . k_{1}=k_{5}=0.48, k_{2}=k_{4}=0.37$, and $k_{3}=0.46$.

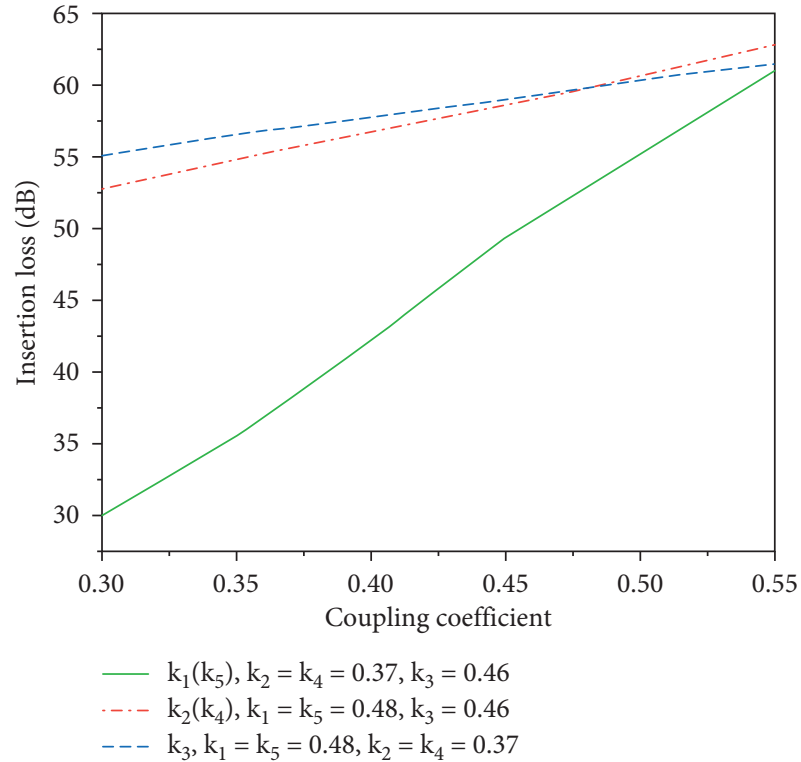

(a)

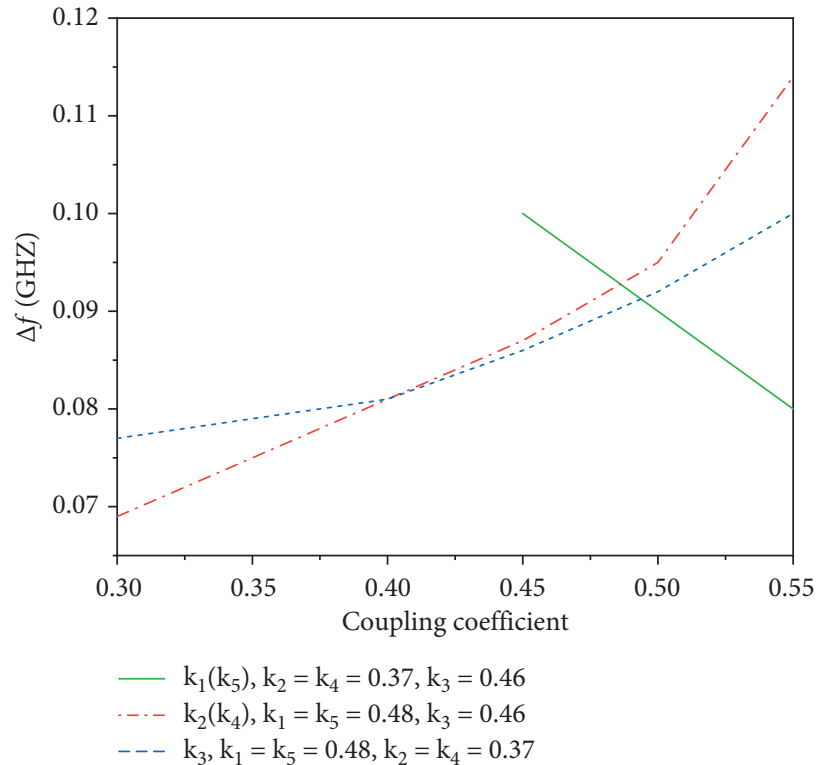

(b)

FIGURE 7: Simulation analysis: (a) the relationship between $k_{1}\left(k_{5}\right), k_{2}\left(k_{4}\right), k_{3}$ and maximum insertion loss; (b) the relationship between $k_{1}\left(k_{5}\right), k_{2}\left(k_{4}\right), k_{3}$ and bandwidth at insertion loss $50 \mathrm{~dB} . Z_{1}=Z_{5}=90 \Omega, Z_{2}=Z_{4}=66 \Omega$, and $Z_{3}=81.3 \Omega$.

\section{External Microstrip Line and PCB Analysis}

In this section, in addition to the width of the external microstrip line, which can carry a current of $10 \mathrm{~A}$, the relationship between the width and the characteristics of $\mathrm{PCB}$ is also discussed. The cross-section structure of PCB is shown in Figure 8, where $M$ is the thickness of the external microstrip line, $H$ is the thickness of the substrate, and $W$ is the width of the external microstrip line. To enable the
MSBSF to be used in high-power transmission lines, the current carrying capacity of the external microstrip line must be considered. According to the IPC-2221standard, when the materials of the external microstrip line and ground plane are copper, the current that an external microstrip line can carry is as follows [25]:

$$
I=0.048 \times d T^{0.44}\left(\frac{W \times M}{(0.0254)^{2}}\right)^{0.725},
$$




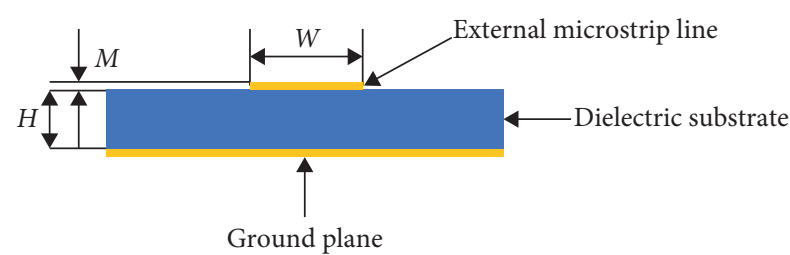

FIGURE 8: The cross-section structure of PCB.

where $I$ is the maximum current and $d T$ is the temperature rise above ambient.

The relationship between the current that an external microstrip line can carry and the width of the line is shown in Figure 9. The higher the current, the larger the width required for the external microstrip line. When the current is $10 \mathrm{~A}$, the width of the external microstrip line is $3.58 \mathrm{~mm}$. Thus, if the external microstrip line carries a current more than $10 \mathrm{~A}$, the width of the line needs to be larger than $3.58 \mathrm{~mm}$.

According to [26-28], when the characteristic impedance of the external microstrip line is a fixed value, the width of the line is related to the dielectric constant and thickness of the substrate. The specific relationship between the width of the external microstrip line and the dielectric constant and the thickness of the substrate is as follows [24]:

$$
Z=\frac{120 \pi}{\sqrt{\varepsilon}}\left(\frac{W}{H}+1.393+0.677 \ln \left(\frac{W}{H}+1.444\right)\right)^{-1}, \frac{W}{H} \geq 1,
$$

where $Z$ is the characteristic impedance of the external microstrip line and $\varepsilon$ is the dielectric constant of the substrate.

To clearly show the relationship between the width of the external microstrip line and the dielectric constant and the thickness of the substrate, the relevant data calculated by line calculation software are shown in Figures 10(a) and 10(b). Figure 10(a) reveals the relationship between the dielectric constant of the substrate and the width of the external microstrip line. With the increase of the dielectric constant of the substrate, the width of the external microstrip line decreases. When the dielectric constant of the substrate is less than 3.1, the width of the external microstrip line is larger than $3.58 \mathrm{~mm}$, and the current flowing through the external microstrip line is greater than 10A. The relationship between the thickness of the substrate and the width of the external microstrip line is plotted in Figure 10(b). With the increase of the substrate thickness, the width of the external microstrip line also increases. When the thickness of the substrate is greater than $1.35 \mathrm{~mm}$, the width of the external microstrip line is greater than $3.58 \mathrm{~mm}$, and the current flowing through the external microstrip line is greater than $10 \mathrm{~A}$.

By analyzing the performance of the external microstrip line passing through the current, it can be found that when the characteristic impedance of the external microstrip line is $50 \Omega$ and the external microstrip line can carry a current of $10 \mathrm{~A}$, the PCB with a substrate thickness of $1.5 \mathrm{~mm}$ and a dielectric constant of the substrate less than 3.1 can be used,

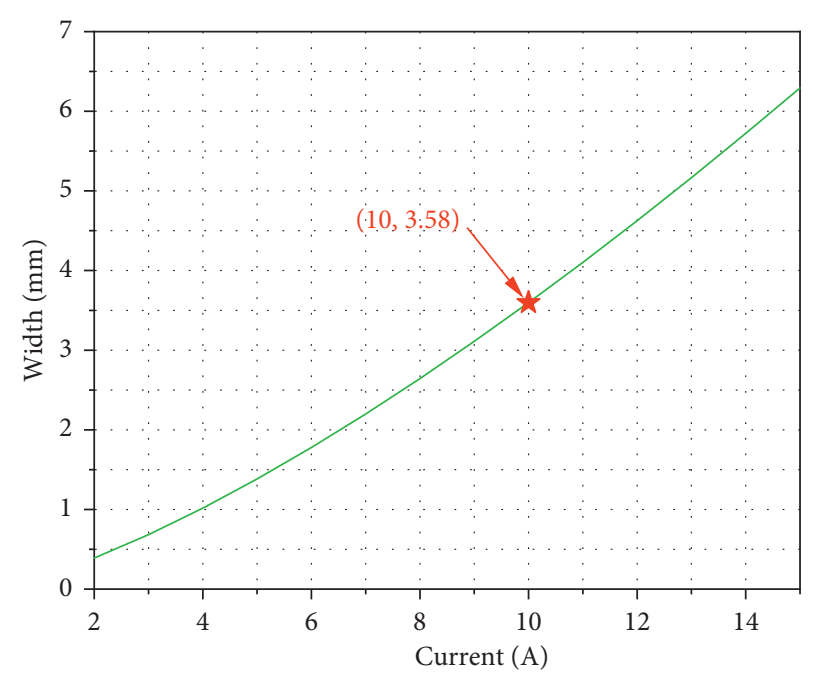

FIGURE 9: The relationship between the current and the width of the external microstrip line $\left(\mathrm{dT}=10^{\circ} \mathrm{C}, M=0.07 \mathrm{~mm}\right)$.

or the PCB with a dielectric constant of 2.65 and a thickness of substrate greater than $1.35 \mathrm{~mm}$ can be used.

Several commonly used PCBs for fabricating MSBSF are shown in Table 1. It can be found that the dielectric constant and substrate thickness of the PCBs meet the requirements, but the cost of F4B is less than that of Rogers.

\section{Experiment and Discussion}

Based on the analysis in Sections 4 and 5, a MSBSF with an insertion loss of $50 \mathrm{~dB}$ in the frequency range of 2.4$2.49 \mathrm{GHz}$ is designed. According to Figures 6 and 7 , $z_{1}=Z_{5}=90 \Omega, \quad Z_{2}=Z_{4}=66 \Omega, \quad Z_{3}=81.3 \Omega, \quad k_{1}=k_{5}=0.48$, $k_{2}=k_{4}=0.37$, and $k_{3}=0.46$. After simulation and optimization by HFSS, the final characteristic impedance parameters of the MSBSF are $Z_{0}=50 \Omega, Z_{1}=Z_{5}=91.33 \Omega$, $Z_{2}=Z_{4}=66.28 \Omega, \quad Z_{3}=81.3 \Omega, \quad Z_{\mathrm{ol}}=Z_{\mathrm{o} 5}=44.1 \Omega$, $Z_{\mathrm{e} 1}=Z_{\mathrm{e} 5}=124 \Omega, \quad Z_{\mathrm{o} 2}=Z_{\mathrm{o} 4}=38.84 \Omega, \quad Z_{\mathrm{e} 2}=Z_{\mathrm{e} 4}=83.74 \Omega$, $Z_{\mathrm{o} 3}=40.24 \Omega$, and $Z_{\mathrm{e} 3}=108 \Omega$. According to Section 5, considering the low cost, the MSBSF is made of F4BM265, and its dielectric constant, thickness of the substrate, and thickness of external microstrip line are $2.65,1.5 \mathrm{~mm}$, and $0.07 \mathrm{~mm}$, respectively. To make the transmission line of the MSBSF pass through the current of $10 \mathrm{~A}$, the unequal width of the coupled structure $[23,29]$ is used. The final layout and the dimensions of the MSBSF are shown in Figure 11(a) and Table 2, respectively. The manufactured MSBSF is presented in Figure 11(b).

To verify the filtering effect of the MSBSF, a vector network analyzer is utilized. The experimental equipment for measuring the filtering effect of the MSBSF is shown in Figures 12(a) and 12(b). The electromagnetic (EM) simulation and measurement results of the MSBSF are plotted in Figure 13. The bandstop filter designed in this paper can produce transmission zeros, but there are no zeros at the center frequency, so the bandwidth of the stopband is narrow. The EM simulation results show that an insertion loss more than $50 \mathrm{~dB}$ can be achieved in the frequency range 


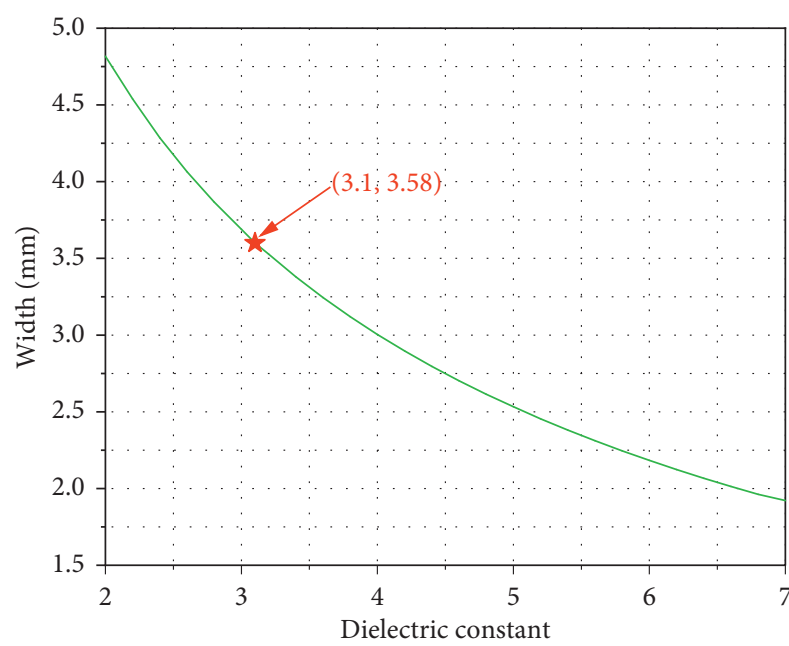

(a)

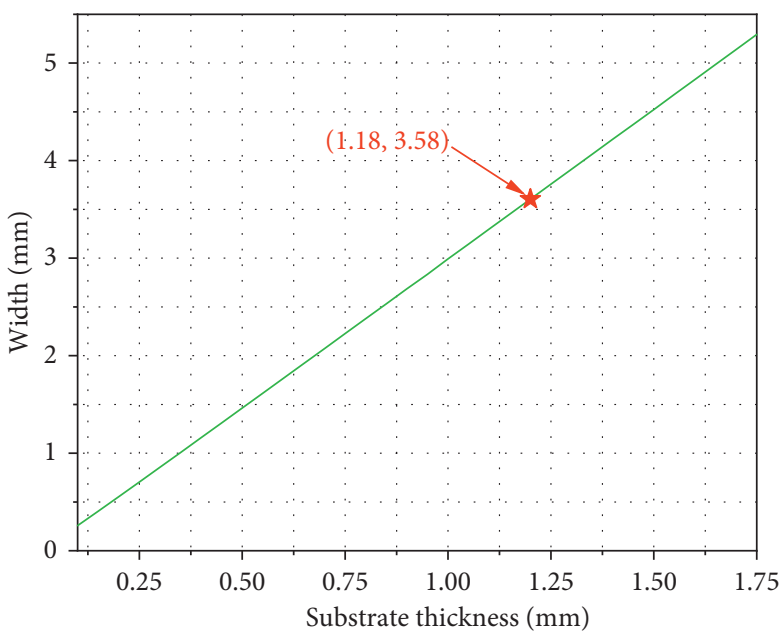

(b)

FIGURE 10: (a) The relationship between the dielectric constant of the substrate and the width of the external microstrip line $(H=1.5 \mathrm{~mm}$, $Z=50 \Omega$ ); (b) the relationship between the thickness of the substrate and the width of the external microstrip line $(\varepsilon=2.65, Z=50 \Omega)$.

TABLE 1: Several commonly used PCBS.

\begin{tabular}{lcccc}
\hline & PCB & & Dielectric constant & Thickness (mm) \\
\hline \multirow{3}{*}{ Rogers } & 5870 & 2.33 & & \\
& 5880 & 2.2 & & \\
& 6002 & 2.94 & & \\
F4B & M220 & 2.2 & & \\
& M255 & 2.55 & $1.5 / 2.0 / 3.0 / 5.0 / 6.0 / 8.0$ & High \\
& M265 & 2.65 & & \\
\hline
\end{tabular}

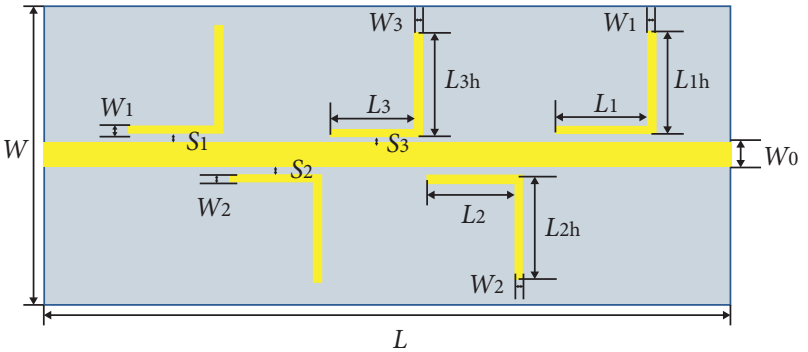

(a)

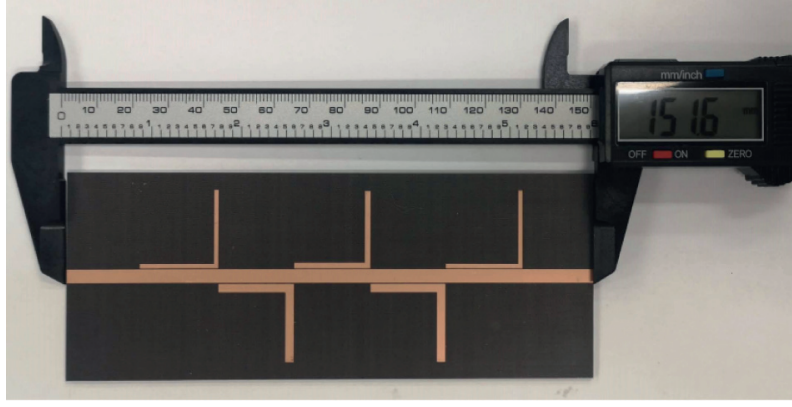

(b)

FIgURE 11: The photograph of the MSBSF. (a) The final layout; (b) the fabricated MSBSF.

TABLE 2: The optimized dimensions of the MSBSF.

\begin{tabular}{lccccccc}
\hline$W_{1}$ & $W_{2}$ & $W_{3}$ & $S_{1}$ & $S_{2}$ & $S_{3}$ & $W$ & $W_{0}$ \\
\hline 1.3 & 2.5 & 1.68 & 0.2 & 0.25 & 0.18 & 59.76 \\
$L_{1}$ & $L_{2}$ & $L_{3}$ & $\mathrm{~L}_{1 \mathrm{~h}}$ & $L_{2 \mathrm{~h}}$ & $L_{3 \mathrm{~h}}$ & $L$ & Unit \\
22.32 & 21.7 & 21.96 & 21.36 & 20.15 & 20.75 & 151.6 & $\mathrm{~mm}$ \\
\hline
\end{tabular}

of $2.40-2.49 \mathrm{GHz}$, and three transmission zeros can be realized in the stopband, which are located at $2.41 \mathrm{GHz}$, $2.44 \mathrm{GHz}$, and $2.48 \mathrm{GHz}$, respectively. Impedance matching must be considered when the microstrip filter is connected to the transmission line to prevent the transmission line conducting electromagnetic information leakage, but impedance matching is not the main research in this paper. Therefore, for matching the impedance, the coaxial 


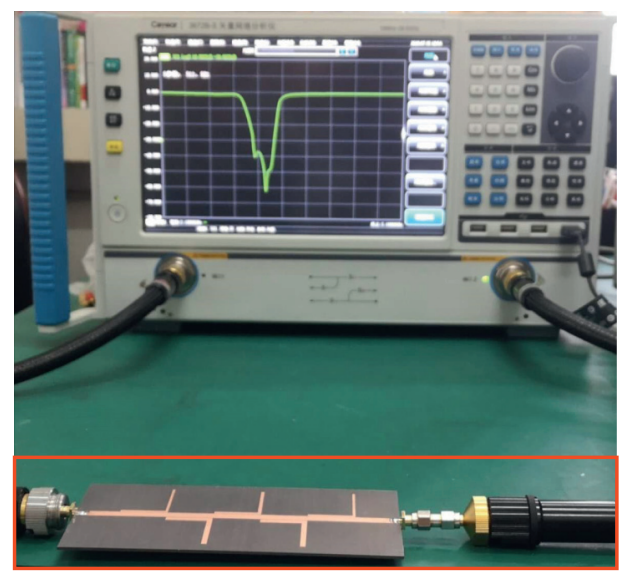

(a)

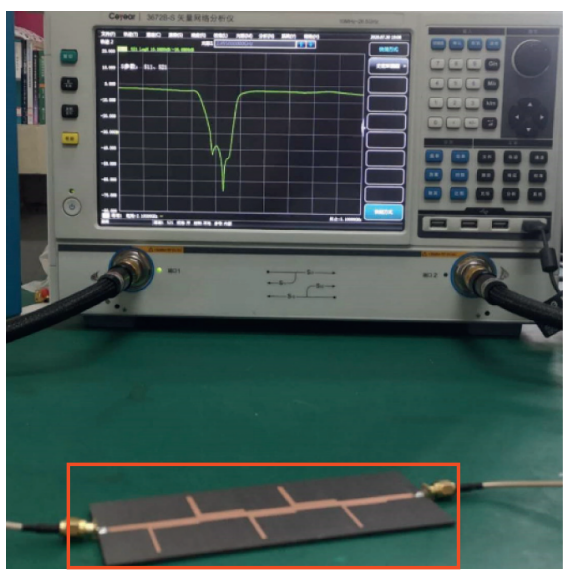

(b)

FIgURE 12: The photograph of experimental equipment for measuring insertion loss: (a) without power line; (b) with power line.

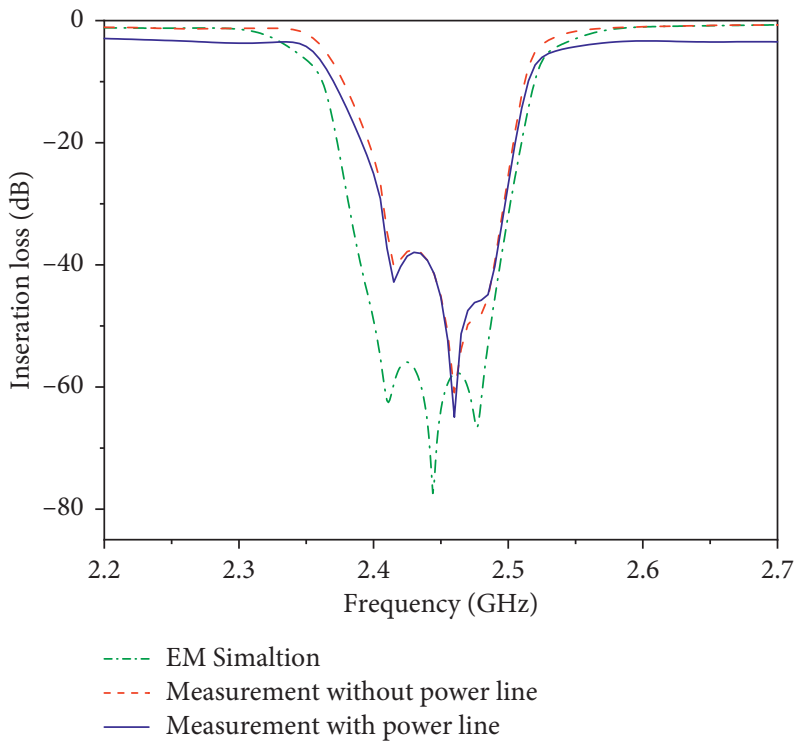

Figure 13: EM simulation and measurement results of the MSBSF.

transmission line is used as the power transmission line. The measurement results of the MSBSFs with and without power transmission line show that the insertion losses of the fabricated MSBSF are all $38 \mathrm{~dB}$, and the frequency ranges are all 2.41-2.49 GHz. The insertion loss and frequency range of measurement with power line and that without power transmission line are in good agreement, but the passband insertion loss measured with power line is larger than that without power line. The results of simulation and measurement are different. The differences between them may come from a tiny dimensional error during the fabrication of MSBSF. The measurement results with and without power transmission line are in good agreement, but there are a few differences. The differences between them may come from the effect of power transmission lines.

To verify that the MSBSF can pass through a current greater than $10 \mathrm{~A}$, a current tester and an electromagnet are used. The current tester provides current, the electromagnet is the load resistance, and the MSBSF is connected in series with the power transmission lines. The experimental equipment for measuring the current is shown in Figure 14(a). It can be found that the MSBSF can carry a current greater than $10 \mathrm{~A}$. To test the withstand voltage performance of the MSBSF, a high-voltage generator is applied. The experimental equipment is shown in Figure 14(b). It can be found that MSBSF can withstand a voltage of $1.7 \mathrm{kV}$.

Through the previously mentioned experiments, it can be found that the MSBSF can achieve an attenuation of $38 \mathrm{~dB}$ to block $2.41-2.49 \mathrm{GHz}$ high-frequency signal and can be used in transmission lines with a power of at least $17544 \mathrm{~W}$.

The comparison among the MSBSF in this paper and the reported filters is provided in Table 3. It can be found that the insertion loss of MSBSF is better than that given in $[19,20]$, and the current and voltage of the filter are analyzed and verified. The comparison between the MSBSF and the EMI 


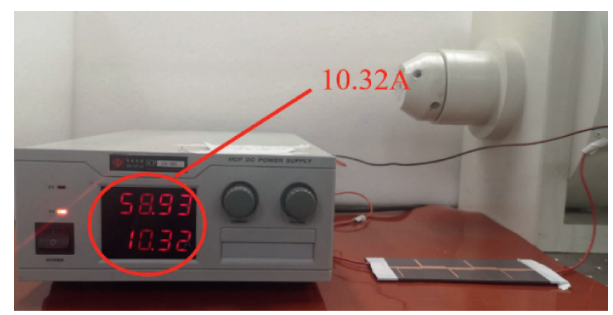

(a)

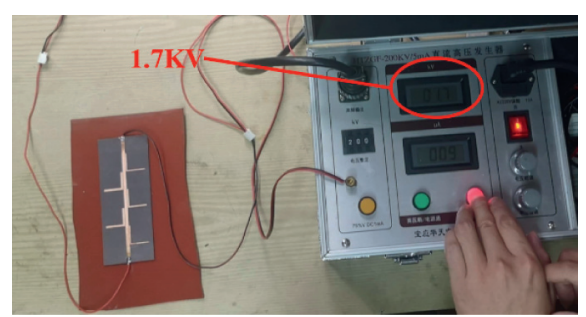

(b)

FIGURE 14: The photograph of experimental equipment: (a) measuring current; (b) measuring voltage.

TABLE 3: Comparison with previously reported filters.

\begin{tabular}{lcccc}
\hline References & Insertion loss $(\mathrm{dB})$ & Frequency range $(\mathrm{GHz})$ & Current $(\mathrm{A})$ & Max voltage $(\mathrm{kV})$ \\
\hline$[19]$ & 30 & $2.2-2.9$ & NA & NA \\
{$[20]$} & 10 & $1.23-5.15$ & NA & NA \\
{$[23]$} & 50 & $2.37-2.65$ & NA & NA \\
This paper & 38 & $2.41-2.49$ & $>10$ & 1.7 \\
\hline
\end{tabular}

NA: the current and voltage of the filters are not analyzed.

TABLe 4: Comparison between MSBSF and EMI filter.

\begin{tabular}{lcccc}
\hline Filter & Frequency range $(\mathrm{Hz})$ & Current $(\mathrm{A})$ & Voltage $(\mathrm{V})$ & Insertion loss $(\mathrm{dB})$ \\
\hline EMI filter & $150 \mathrm{~K}-30 \mathrm{M}$ & 10 & 250 & 50 \\
MSBSF & $2.41 \mathrm{G}-2.49 \mathrm{G}$ & $>10$ & $1.7 \mathrm{~K}$ & 38 \\
\hline
\end{tabular}

filter is provided in Table 4. It can be found that the MSBSF withstands higher voltage and carries greater current than EMI filters in addition to being used in high-frequency bands.

\section{Conclusion}

In this paper, a high insertion loss microstrip bandstop filter (MSBSF), which can be applied to high-power transmission lines, is designed. According to the structure of the traditional branch load band stop filter, the equivalent structure and the unequal-width technology of the parallel coupled microstrip line are used to realize the filter can carry large current and have high insertion loss by increasing the filter order. The MSBSF is composed of $\mathrm{n}$ parts, and each part consists of a parallel coupling line with an open stub. To produce the MSBSF with a high insertion loss, an analysis is carried out, and it is found that the MSBSF consisting of five parts can realize an insertion loss more than $40 \mathrm{~dB}$ and has a simple structure. Then, the MSBSF is analyzed from both theory and simulation aspects, and the design process is summarized. Besides, the width of the external microstrip line which can carry a current of $10 \mathrm{~A}$ is analyzed, and the characteristics of PCB which can carry a current of $10 \mathrm{~A}$ are summarized. Finally, according to the design process of MSBSF, the requirements of the external microstrip line carrying a current of $10 \mathrm{~A}$, and the characteristics of PCB which can carry a current of $10 \mathrm{~A}$, MSBSF with an insertion loss of $50 \mathrm{~dB}$ in the frequency range of $2.40-2.49 \mathrm{GHz}$ is designed and fabricated with low cost F4BM265. To test the performance of MSBSF, a vector network analyzer, a current tester, and a high-voltage generator are used. It is found that the attenuation of MSBSF is $38 \mathrm{~dB}$ at $2.412 .49 \mathrm{GHz}$, and the MSBSF can carry a current greater than $10 \mathrm{~A}$ and withstand the voltage of $1.7 \mathrm{kV}$. Hence, the designed MSBSF may be used in preventing high-frequency conduction electromagnetic information leakage of high-power transmission lines. Microstrip filter has good performance in microwave frequency band, and because of its unique structure, it is convenient to connect with transmission line and has unique advantages in preventing transmission line conduction electromagnetic information leakage. In order to better filter electromagnetic information in transmission line, the filter used to prevent transmission line conduction electromagnetic information leakage should have high insertion loss. Therefore, the research of filter with high insertion loss has research value in preventing transmission line conduction electromagnetic information leakage and should become one of the performance improvements of band stop filter in the future. Although the range of work frequency of the filter that we discuss in this paper is very narrow $(2.41-2.49 \mathrm{GHz})$, it is a part of our current research work. The range of work frequency of this filter has the possibility of broadening, but it requires further simulation and verification, which is also what we will do in the future.

\section{Data Availability}

The data used to support the findings of this study are included within the article.

\section{Conflicts of Interest}

The authors declare no conflicts of interest. 


\section{Authors' Contributions}

Xueyun Han is responsible for conceptualization and methodology and funding acquisition. Pengfei Zheng was responsible for software, validation, formal analysis, and original draft preparation. Xiaosong Li was responsible for review and editing. Yingping Zhou was responsible for project administration. Xueyun Han and Pengfei Zheng contributed equally to this work. All authors have read and agreed to the published version of the manuscript.

\section{Acknowledgments}

The Key Discipline Open Lab of Electromagnetic Wave Characteristic Information Detection of Henan Province provided the equipment. This work was supported by the Key Projects of Science and Technology of Henan Province (Grant nos. 212102210218 and 202102210316) and Foundation of Henan Educational Committee (Grant nos. 22A510007 and 22A430025).

\section{References}

[1] S. Rooney, J. E. van der Merwe, S. A. Crosby, and I. M. Leslie, "The Tempest: a framework for safe, resource assured, programmable networks," IEEE Communications Magazine, vol. 36, no. 10, pp. 42-53, 1998.

[2] B. B. Yilmaz, M. Prvulovic, and A. Zajic, "Electromagnetic side channel information leakage created by execution of series of instructions in a computer processor," IEEE Transactions on Information Forensics and Security, vol. 15, pp. 776-789, 2020.

[3] H. Sekiguchi and S. Seto, "Study on maximum receivable distance for radiated emission of information technology equipment causing information leakage," IEEE Transactions on Electromagnetic Compatibility, vol. 55, no. 3, pp. 547-554, 2013.

[4] W. Takashi, N. Hiroto, and S. Hiroshi, "A display technique for preventing electromagnetic eavesdropping using color mixture characteristic of human eyes," Information Hiding, vol. 5284, pp. 1-14, 2008.

[5] H. S. Lee, D. H. Choi, K. Sim, and J.-G. Yook, "Information recovery using electromagnetic emanations from display devices under realistic environment," IEEE Transactions on Electromagnetic Compatibility, vol. 61, no. 4, pp. 1098-1106, 2019.

[6] C. Kasmi, M. Hélier, M. Darces, and E. Prouff, "Modeling extreme values resulting from compromising electromagnetic emanations generated by an information system," Comptes Rendus Physique, vol. 15, no. 5, pp. 415-420, 2014.

[7] V. Yli-Mayry, D Miyata, N. Homma, T. Aoki, and Y. Hayashi, "On the evaluation of electromagnetic information leakage from mobile device screens," in Proceeding of the IEEE International Symposium on Electromagnetic Compatibility and the IEEE Asia-Pacific Symposium on Electromagnetic Compatibility, pp. 1050-1052, Singapore, May 2018.

[8] I. Kubiak, "Influence of the method of colors on levels of electromagnetic emissions from video standards," IEEE Transactions on Electromagnetic Compatibility, vol. 61, no. 4, pp. 1129-1137, 2019.

[9] I. Kubiak, A. Boitan, and S. Halunga, "Assessing the security of TEMPEST fonts against electromagnetic eavesdropping by using different specialized receivers," Applied Sciences, vol. 10, no. 8, p. 2828, 2020.

[10] I. Kubiak, "Impact of IT devices production quality on the level of protection of processed information against the electromagnetic infiltration process," Electronics, vol. 8, no. 9, p. 1054, 2019.

[11] T. L. Song, Y. R. Jeong, H. S. Jo, and J. G. Yook, "Noisejamming effect as a countermeasure against TEMPEST during high-speed signaling," IEEE Transactions on Electromagnetic Compatibility, vol. 57, pp. 1491-1500, 2005.

[12] S. Yoneda, Y. Shiraki, Y. Sasaki, and C. Miyazaki, "A gasketfree electromagnetic shielding structure for 2.4-GHz and 5$\mathrm{GHz}$ bands using cascaded dual-behavior SIW resonators," IEEE Transactions on Electromagnetic Compatibility, vol. 62, no. 2, pp. 377-385, 2020.

[13] Y.-I. Hayashi, N. Homma, T. Mizuki et al., "Analysis of electromagnetic information leakage from cryptographic devices with different physical structures," IEEE Transactions on Electromagnetic Compatibility, vol. 55, no. 3, pp. 571-580, 2013.

[14] H. S. Efendioglu, M. Yabuloglu, and H. Ozer, "Effectiveness of shielded window films used for electromagnetic leakages in communication security," in Proceeding of the IEEE IV International Electromagnetic Compatibility Conference, pp. 14, Ankara, Turkiye, September 2017.

[15] S. Jeong, D. Shin, and J. Kim, "A transformer-isolated common-mode active EMI filter without additional components on power lines," IEEE Transactions on Power Electronics, vol. 34, no. 3, pp. 2244-2257, 2019.

[16] J. Loughry, "Optical TEMPEST," in Proceeding of the International Symposium on Electromagnetic, pp. 172-177, Amsterdam, Netherlands, August 2018.

[17] K. Shaneman and S. Gray, "Optical network security: technical analysis of fiber tapping mechanisms and methods for detection \& prevention," in Proceeding of the IEEE Military Communications Conference, pp. 711-716, Monterey, CA, USA, November 2004.

[18] K. Hoffmann and Z. Skvor, "First results of microwave measurement of broadband radio interference filters," in Proceeding of the 64th ARFTG Microwave Measurements Conference, pp. 139-142, Orlando, FL, USA, December 2004.

[19] K. Song, F. Zhang, M. Fan, Y. Zhu, and Y. Fan, "Compact broadband bandstop filter based on composite right/left handed transmission line," Electromagnetics, vol. 37, no. 4, pp. 196-202, 2017.

[20] C.-K. Chan, C.-H. Cheng, and T.-L. Wu, "Design of a broadband common-mode filter with four transmission zeros," IEEE Transactions on Electromagnetic Compatibility, vol. 61, no. 4, pp. 1052-1060, 2019.

[21] P. Phudpong and I. C. Hunter, "Nonlinear matched reflection mode bandstop filters for frequency selective limiting applications," in Proceeding of the IEEE/MTT-S International Microwave Symposium, pp. 1043-1046, Honolulu, USA, June 2007.

[22] W. J. Feng, W. Q. Che, S. Y. Shi, and Q. Xue, "Compact dualwideband bandstop filters based on open-coupled lines and transversal signal-interaction concepts," IET Microwaves, Antennas \& Propagation, vol. 7, no. 2, pp. 92-97, 2013.

[23] C.-H. Wu, C.-C. Tang, and C.-W. Tang, "Design of the microstrip bandstop filter with high insertion loss," IEEE Transactions on Components, Packaging, and Manufacturing Technology, vol. 9, no. 1, pp. 122-128, 2019.

[24] J. S. Hong, Microstrip Filters for RF/Microwave for Applicationpp. 6-10, John Wiley \& Sons, Canada: New jersey, 2nd ed. edition, 2011. 
[25] J. Adam, "New correlations between electrical current and temperature rise in PCB traces," in Proceeding of IEEE Semiconductor Thermal Measurement and Management Symposium, pp. 292-299, San Jose, CA, USA, March 2004.

[26] W. J. Getsinger, "Measurement and modeling of the apparent characteristic impedance of microstrip," IEEE Transactions on Microwave Theory and Techniques, vol. 31, no. 8, pp. 624-632, 1983.

[27] M. Kobayashi, "Frequency dependent characteristics of microstrips on anisotropic substrates," IEEE Transactions on Microwave Theory and Techniques, vol. 30, no. 11, pp. 20542057, 1982.

[28] M. Kirschning and R. H. Jansen, "Accurate model for effective dielectric constant of microstrip with validity up to millimetre-wave frequencies," Electronics Letters, vol. 18, no. 6, pp. 272-273, 1982.

[29] C.-W. Tang, C.-T. Tseng, and S.-C. Chang, "Design of the compact tunable filter with modified coupled lines," IEEE Transactions on Components, Packaging, and Manufacturing Technology, vol. 4, no. 11, pp. 1815-1821, 2014. 